\title{
Food Engineering of Meat Bio-Products to Immunity Strengthening
}

\author{
Julia Bazarnova, Aleksey Korzh, Natalya Barsukova*, Svetlana Eliseeva and Ekaterina \\ Fedinishina
}

Graduate School of Biotechnology and Food Science, Institute of Biomedical Systems and Biotechnology, Peter the Great St.Petersburg Polytechnic University, 195251 Polytechnicheskaya 29, St. Petersburg, Russia

\begin{abstract}
The mainstream of healthy nutrition during the new coronavirus pandemic is adequate and balanced nutrition based on strengthening human immunity. The authors consider the main aspects of developing theory and practice of adequate nutrition using innovative biotechnologies. Based on the St. Petersburg startup GASTROMAN.LAB there was developed new line of Bio-products enriched with probiotic cultures of microorganisms, biologically active substances of plants and prebiotics that was implemented using modern technological methods of food engineering. The recipes of original meat products of the Petersburg cuisine were used as basis for new developed recipes of bio-culinary products.
\end{abstract}

\section{Introduction}

Food engineering is an interdisciplinary field of applied physical sciences, which includes the research, production and use of enzymes, viruses, microorganisms, cell cultures of animals and plants, as well as products of their biosynthesis and biotransformation in the food industry.

The COVID-19 pandemic showed that public health measures to prevent infectious diseases include boosting immunity through improved nutrition. Immune dysfunction is now considered a cause and a consequence of poor nutrition.

According to marketing research by ADM [1], the pandemic has prompted consumers to take an active interest in foods that are beneficial to immunity. For example, about $80 \%$ of consumers surveyed believe probiotics are beneficial for maintaining a healthy digestive system and strengthening immunity. Research conducted in 2020 found that 6 out of 10 shoppers surveyed were more likely to look for immune-boosting products on store shelves, with one in three respondents indicating that these concerns in consumer behavior have intensified since fall 2020. Experts predict that this trend to be sustainable and continued in 2021. According to a global study of the market for foods that stimulate immunity, conducted by the Business Research Company, in the coming years, the world market for this category

\footnotetext{
* Corresponding author: barsukova_nv@spbstu.ru
} 
of foods is expected to grow from the current 830 billion dollars per year to 1 trillion dollars by 2023 [2].

The aim of our research is to study modern trends in the field of food engineering aimed at creating products to strengthen immunity, and the development of bio-products enriched with probiotic cultures of microorganisms and prebiotics, using modern technological methods of food engineering.

\section{Research objects and methods}

As research objects were used pro- and prebiotics used in the food industry; innovative food technologies; meat products enriched with pro- and prebiotics.

The choice of functional ingredients and methods of technological processing of meat to create healthy food products that strengthen the immune system, was carried out on the basis of an analytical review of the literature and generalization of the authors' own practical experience in the development of recipe composition of meat products.

The experimental part consisted in the development of original recipes and technologies for culinary products from meat, including functional additives of probiotic cultures and dietary fiber (prebiotics).

\section{Results and Discussion}

In the food industry there is currently a paradigm shift towards developing food products with additional consumer value associated with their benefits for strengthening immunity and maintaining human health. Food manufacturers are actively rethinking strategies and investing heavily in food engineering startups to find and implement a scalable food business model aimed at boosting immunity and improving wellness through nutrition. One notable example is Foodini's new robotic machine, which uses a 3D printer to "print" fortified foods made from natural organic raw materials and healthy micronutrients. Universitat de Barcelona launched an innovative startup, BeYou, specializing in the development of digital solutions to promote healthy habits that strengthen the immune system through personalized nutrition.

Analysts following the changes in the global food market believe that the growing interest of consumers in their health has led to an expansion of the list of consumer properties of products. This fact may be associated with the development of food engineering in the field of using healthy ingredients. Among the latter, it is worth highlighting the ingredients with pronounced antiviral activity, including turmeric, cinnamon, moringa, elderberry, cordyceps mushroom extract, tulasi leaves (thin-colored Indian basil) and other useful plants.

Since ancient times, herbs have been popular ingredients in recipes of many meat products. Some of them are immunostimulants, some are capable of activating nonspecific defense mechanisms of the human body against the spread of viruses and pathogenic microorganisms that cause infectious diseases. For example, the leaves of tulasi (Basil thinflowered, Latin Ocimum tenuiflorum) contain oleanolic, ursolic, rosmarinic acids, eugenol, carvacrol, menalool, caryophyllene and other phytochemicals that have antiviral and antioxidant activity, which help strengthen the immune system. For example, the use of an extract of the Cordyceps mushroom containing the active ingredient Cordycepin, which has antiviral properties, helps prevent viral diseases and strengthening immunity.

The study of the role of carotenoids in antioxidant regulation, gene regulation, and apoptosis made it possible to deepen knowledge about the mechanisms of strengthening the immune function [3]. The authors report that hesperidin, contained in the peel of oranges, promotes the growth and development of lactobacilli, enterococci, staphylococci, inhibits the 
development of bacteria of the genus Clostridium and Eubacterium, promotes immune modulation and upregulation of immunoglobulin A and lymphocytes [4].

The results of recent studies by A. M. Valdes indicate that low consumption of fiber, pectin and other prebiotic fibers reduces the biosynthesis of short-chain fatty acids and disrupts the metabolism of the intestinal microbiota, leading to the accumulation of potentially harmful metabolites [5]. It is known that low fiber content in diets leads to the destruction of the biological barriers of the colon mucosa, causing inflammation [6].

The author of the theory of adequate nutrition, A.M. Ugolev, found that dietary fiber plays a significant role in the normalization of the gastrointestinal tract (especially the small and large intestine), increases the mass of the muscle layer, affects its motor activity, the rate of absorption of nutrients in the thin intestine, pressure in the cavity of the digestive apparatus, electrolyte metabolism in the body, etc. $[7,8]$. It has been found that dietary fiber, including polysaccharides and lignin, has the ability to bind water and bile acids, as well as absorb toxins. The ability of plant fiber to bind water has a significant effect on the rate of transit of the contents of the gastrointestinal tract. Fiber in bran binds 5 times more water than their own weight, and ultra-grained fibers of carrots or turnips - 30 times more.

It is widely known that dietary fiber affects the habitat of bacteria in the human intestine and strengthens the immune system. For example, in 2020, a study by De Marco Castro et al. examined the immunomodulatory mechanisms of action of prebiotics beta-glucans. Betaglucans have been found to "train" the immune cells of the human body, causing changes that help fight infections of the upper respiratory tract and reduce the risk of developing respiratory diseases [9]. Observations indicate that the immunostimulatory effects of prebiotics are associated with the metabolism of probiotic microorganisms and their production of short-chain branched-chain fatty acids (Table 1).

Table 1. Influence of prebiotic fibers on strengthening human immunity.

\begin{tabular}{|l|l|}
\hline \multicolumn{1}{|c|}{ Prebiotics } & \multicolumn{1}{|c|}{ Influence on strengthening the immune system } \\
\hline Cellulose & $\begin{array}{l}\text { Promotes the growth of bacteria of the genus Prevotella, producing short- } \\
\text { chain fatty acids. }\end{array}$ \\
\hline Inulin & $\begin{array}{l}\text { Promotes the growth and development of Bacillus coagulans, Bacillus } \\
\text { subtilis, Faecalibacterium prausnitzii, Bacteroides fragilis, } \\
\text { bifidobacteria, lactobacilli and bacteria that produce butyrate, acetate, } \\
\text { propionate. Increases the barrier function of the intestinal mucosa. }\end{array}$ \\
\hline Pectin & $\begin{array}{l}\text { Promotes the growth and development of Bifidobacterium odolescentis, } \\
\text { Ruminococcus bromii, Eubacterium rectale. }\end{array}$ \\
\hline Resistant starch & $\begin{array}{l}\text { Promotes the growth of bacteria of the genus Firmicutes and } \\
\text { bifidobacteria. Reduces the content of potentially carcinogenic secondary } \\
\text { bile acids. Helps reduce abdominal fat. }\end{array}$ \\
\hline Kefiran & $\begin{array}{l}\text { Possesses powerful antitumor, immunomodulatory activity, inhibits the } \\
\text { synthesis of allergens (histamine), lowers the level of bad cholesterol in } \\
\text { the blood. }\end{array}$ \\
\hline Beta glucans & $\begin{array}{l}\text { Promote the growth and development of bifidobacteria, lactobacilli, } \\
\text { bacteroids. Reduces the risk of developing respiratory diseases. }\end{array}$ \\
\hline Arabinogalactan & $\begin{array}{l}\text { Stimulates the growth and development of Coprococcus, } \\
\text { Lachnoclostridium, Bifidobacterium }\end{array}$ \\
\hline Arabinoxylan & \multicolumn{1}{|c}{ on } \\
\hline
\end{tabular}

1. Various probiotic cultures are used for the production of fermented foods. Intaking of food and culinary products containing probiotics improves the phagocytic activity of granulocytes, which increases the natural immunity of a person $[10,11,12]$. It is known that a healthy human intestine contains a huge number of so-called "friendly" microorganisms: bifidobacteria, lactobacilli, propionic acid bacteria and other symbiotic cultures that make up 
the human intestinal microbiome. These beneficial microorganisms living on the intestinal walls actively synthesize special substances - lymphocytes - on the surface of Peyer's plaques. Lymphocytes, penetrating through the intestinal walls into the human lymphatic system, actively produce immunoglobulins (lysozyme, lactoferrin, phagocytes, interferons), which are immune factors that strengthen the biological immunity of a person. A decrease in the concentration of beneficial microflora in the intestine entails an increase in the body's vulnerability to pathogens (E. coli, Shigella, some species of Streptococcus, etc.) due to a decrease in the Shannon biodiversity index and, as a result, leads to a decrease in immunity.

Probiotics improve the barrier function of the epithelium and strengthen the intestinal wall by reducing its permeability, helping to reduce the risk of inflammatory processes in the intestine and oxidative damage to the liver. For example, studies in Australia have shown that the Bacillus coagulans GBI-30 strain has beneficial effects on digestion and is able to modulate the immune response in upper respiratory tract infections. Lactobacillus casei 431 strain is able to activate immune cells, increase the production of immune cell precursors and increase the secretion of antibodies. Some probiotics demonstrate a pronounced ability to increase antibody secretion in order to improve protection against pathogenic invasions and thus can serve as vaccine adjuvants [13].

The results of numerous studies indicate that in many cases the progress of noncommunicable diseases is directly related to disorders of the gut microbiome. The human gut microbiome is now recognized as the most important biological interface between genotype, environment and lifestyle. Nutritional recommendations based on in-depth analysis of the human genome and microbiome, as well as information from biomarkers and biochemical research, are growing in popularity.

2. For the production of new generation food products that contribute to strengthening human immunity, new techniques and technological practices of food engineering are currently used, which combine applied biotechnology with innovative vacuum, cavitation, additive and other processes and technological methods of molecular gastronomy [14, 15].

To enrich dietary meat products with useful micronutrients and increase their juiciness, we used the technology of vacuum processing of the cookvac product. The process of marinating meat was carried out in a cookvac apparatus [16]. Semi-finished meat products were put into the apparatus, filled with various marinades (fruit, berry, classic, based on herbs and nuts, etc.), evacuated until the required pressure was established (0.25-0.5-0.75-0.9 bar) and left to marinate for different times (10-15-20-30 minutes). Next, the resulting meat products were subjected to heat treatment in a cookvac apparatus. As a result of the research, the optimal pressure and marinating time for meat products was established. The lowest heat loss was observed with turkey meat products marinated at 0.5 bar for 20 minutes. The culinary products obtained using the cookvac technology differed from traditional ones in taste and aroma, as well as juiciness and texture characteristics. The greatest juiciness was found in portioned meat products from turkey in a sweet and sour marinade based on cranberries, orange juice, pineapple, turmeric, garlic and red pepper [17].

At the production site of the GASTROMAN.LAB startup, the creator of which is the author of the article A. Korzh, healthy meat Bio-snacks were developed, made on the basis of improved recipes of authentic meat products of St. Petersburg cuisine, with addition of probiotic cultures and dietary fiber:

- Bio-fromage from poultry with the addition of a complex probiotic-producer of butyrate, based on a consortium of microorganisms Clostridium butyricum, Bacillus subtillis, Enterococcus faecium and Jerusalem artichoke powder obtained by innovative thermomixing technology;

- Bio-galantin from poultry in jelly with the addition of the probiotic Lactobacillus rhamnosus in the jelly, which, in addition to having a positive effect on the intestines and 
enhancing the immune response, has a powerful anti-infectious, anticarcinogenic and antiallergic effect;

- Bio-«chaud-froid» from poultry with the addition of the prebiotic lactulose and "Bifiton" starter culture

- Bio-pate from the liver in a dough, with the addition of turmeric and the complex prebiotic Dubinin, containing in its composition fiber, inulin, arabinoxylan and other prebiotic fibers and obtained using the technology of ultrasonic homogenization;

- Cervelas with the addition of the useful polysaccharide kefiran, obtained by ultrasonic processing of kefir.

To extend the shelf life of the developed cold meat Bio-snacks, new methods of introducing pure probiotic cultures are recommended for use, including spraying on the surface of finished meat products before packaging them, introducing probiotic cultures into a cold creamy jelly sauce, andob when making cold meat Bio-snack.

\section{Conclusion}

The conclusions obtained during the implementation of the GASTROMAN.LAB startup under the scientific guidance of the Graduate School of Biotechnology and Food Science indicate that for the successful implementation of a scalable business model for the production of new generation products and their promotion on the market, a comprehensive understanding of the mechanisms of psychological perception by the consumer of Bioproducts and culinary meat products and the popularization of healthy nutrition among the population, which is undoubtedly an important task of the development of society.

\section{References}

1. Top Five Global Trends that Will Shape the Food Industry in 2021 (2020) https://www.businesswire.com/news/home/20201027005626/en/. Accessed 11 January 2021

2. Global Immunity Boosting Food Products Market https://www.thebusinessresearchcompany.com/report/immunity-boosting-foodproducts-market. Accessed 20 January 2021

3. B.P. Chew, J.S. Park, The Journal of nutrition 134(1) (2004)

4. S. Estruel-Amades, M. Massot-Cladera, F.J. Pener-Cano, A. Franch, M. Castell, M. Camps-Bossacoma, Naturients 11(2) (2019)

5. A.M.Valdes, J.Walter, E.Segal, T.D.Spector, BMJ 361 (2018)

6. A. Almeida, A.L. Mitchell, M. Boland, S.C. Forster, G.B. Gloor, A. Tarkowska, T.D. Lawley, R.D. Finn, Nature 568 (2019)

7. A.M. Ugolev, Teoriya adekvatnogo pitaniya i trofologiya (Nauka, Leningrad,1991)

8. A.M. Ugolev, Estestvennye tekhnologii biologicheskih system (Nauka, Leningrad,1987)

9. E. De Marco Castro, P.C. Calder, H.M. Roche, Mol. Nutr. Food Res. 65 (2021)

10. V. Sangwan, S.K. Tomar, R.R.B. Singh, A.K. Singh, B. Ali., Journal of food science 76 (4) (2011)

11. B.A. Shenderov, Gastroenterologiya Sankt-Peterburga 2-3 (2010)

12. B.A. Shenderov, Vestnik vosstanovitel'noj mediciny 4 (2017)

13. L. Vitetta, E. T. Saltzman, M. Thomsen, T. Nikov, S. Hall, Vaccines 5(4) (2017) 
14. J. Bazarnova, S. Eliseeva, N. Zhilinskaya, N. Barsukova, E. Aronova, A. Korzh, ICEPP-2020 E3S Web of Conferences 161 (2020)

15. N. Myhrvold, C. Young, M. Bilet, Modernist Cuisine: The Art and Science of Cooking (The Cooking Lab, 2011)

16. Cookvac- a new word in the gastronomic restaurant kitchen https://pitportal.ru/vestnik/section157/9036.html Accessed 31 January 2021

17. E.V. Moskvicheva, N.V. Barsukova, I.V. Ugryumov, S.A. Okunevich, R.D. Deniskin, Meat industry 7 (2017) 